\title{
New Mobility Scheme for Reducing Location Traffic in Mobile Networks
}

\author{
Il-Sun Hwang ${ }^{1}$, Myungsun Lee ${ }^{1}$, Ki-sung Yoo ${ }^{1}$, and Jin-wook Chung ${ }^{2}$ \\ 1 R\&D Network Management KISTI, Korea \\ his@kisti.re.kr \\ 2 Dept. of Computer Eng. SungKyunKwan Univ., Korea
}

\begin{abstract}
A major problem in such a mobile networks is how to locate mobile hosts. Two major operations, the location registration and the call tracking, are involved in managing a mobile host's location. The previous works can only minimize the traffic cost of one of these two operations. In this paper we propose new strategy that minimize the costs of both operations. Our performance analysis proves that the proposed strategy is superior to the previous methods.
\end{abstract}

\section{Introduction}

A great difference between wireless network environment and traditional wired communication environment us that it allows users to migrate anywhere and to retrieve all kinds of data they want anytime in mobile computing environment. To effectively monitor the movement of each mobile client, a large geographical region is partitioned into small Registration Areas (RA). Each RA has a Mobile Switching Center (MSC)which serves as the local processing center of the RA. The profiles of mobile clients inside a RA are kept, in the MSC's Visitor Location Register (VLR). On top of several MSC/VLRs is a Local signaling Transfer Point (LSTP) and on top of several LSTPs again is a Remote Signaling Transfer Point (RSTP). In this way, the whole system forms a hierarchy of station. The LSTP and the RSTP are routers for handling message transfer between stations. For one RSTP there is a Home Location Register (HLR). Each mobile client must register in a HLR.Communication cost depends on how high the message has to go up the hierarchy, as it indicates the distance and routers it has to go through.

In spite of many advantages available in wireless communication. It is not without difficulties to realize such systems. The first problem is how to locate a mobile user in a wireless environment. The IS-41 standard strategy is most often referred in resolving this problem. IS-41 used in the United States and GMS [6] used in Europe are examples of this strategy. Many papers in the literature have demonstrated that the IS-41 strategy does not perform well. This is mainly because whenever a mobile client moves. The VLR of a RA which detected the arrival of the client always reports to the HLR about the client's new location. Several other location management strategies have been proposed to improve the performance of the IS-41 strategy. Among them, the Forwarding strategy 
[4] [5] the Local Anchor (LA) strategy [8], and the Caching strategy [11] are representatives of the old VLR to the new VLR. Update of the client's location to the HLR's database is not always needed to minimize communications to the HLR. To locate a callee however, some extra time is required to follow the forwarding link(s) to locate the client. When the number of the forwarding links is high, the locating cost becomes significant. Our strategy, termed the Dynamic Cache \& Carry (DCC) strategy, tries to further improve the performance of these previous methods by minimizing the locating cost. The key idea is for each mobile client (1) we define the VLRs that have been linked to by the same LA as the overseen VLRs of this LA, (2) a link from the overseen VLR to its LA is cached in the VLR, and (3) we allow multiple such LAs to be linked together by using forwarding links. In order to computer the performance improvement over the previous methods can be achieved.

\section{The IS-41 Strategy}

According to the IS-41 strategy, the HLR always knows exactly the ID of the serving VLR of a mobile terminal. We outline the major steps of the IS-41 location registration scheme as follows $[6]$ :

1. The mobile terminal sends a registrationrequest(REGREQ) message to the new VLR.

2. The new VLR checks whether the terminalis already registered. If not, it sends a registration notification (REGNOT) message to the HLR

3. The HLR sends a registration cancellation (REGCANC) message to the old VLR.

The old VLR deletes the information of the terminal and the IS-41 call tracking scheme is outlined as follows:

1. The VLR of caller is queried for the information of callee. If the callee is registered to the VLR, the SEARCH process is over and the call is established. If not,the VLR sends a location Request (LOCREQ) message to the HLR.

2. The HLR finds out to which VLR the callee is registered, and sends a routing request (ROUTREQ) message to the VLR serving the callee. The VLR finds out the location information of the callee.

3. The serving MSC assigns a temporary local directory numbers(TLDN) and returns the digits to the VLR which sends it to the HLR.

4. The HLR sends the TLDN to the MSC of the caller.

5. The MSC of the caller establishes a call by using the TLDN to the MSC of the callee. Among the above 5 steps, the SEARCH process is composed of step1 and step2.

\section{DCC Strategy}

The previous methods for improving the IS-41 mechanism were all designed to reduce the locating cost. 


\subsection{Location Registration}

The basic idea of the DCC strategy is that the new VLR determines whether reports to the LA or the HLR the mobile client's new location when a mobile client comes into its territory. Figure 1 tells how the new VLR reports to the HLR about the mobile client's new location. How the new VLR informs the HLR is new in this strategy. How the new VLR informs the HLR is new in this strategy. The algorithm is as follows.

1. When the new VLR learns that a mobile client is coming into its territory, it will determine whether requesting a location update to the LA or the HLR. If the new location of the mobile client is outside the area that the LSTP of the LA controls (before the mobile client moves to the new VLR), jump to the Step 7 (the new VLR will request a location update to the HLR). Otherwise, go on to the next step (the new VLR informs the LA of location Update).

2. The old VLR replies an acknowledgement to the new VLR.

3. The old VLR informs the LA that the mobile client has moved to the new VLR. Also the old VLR update its own MCL table by replacing the mobile client's Type value with "Visited VLR" and the Pointer value with the LA's ;location.

4. The LA replies a message to the old VLR, and updates its own MCL table. The Type value of the mobile client is not changed. The Pointer value is modified to the new VLR's location.

5. End of the Location Registration.

6. Goto Step 13.

7. The new VLR informs the HLR of the arrival of the mobile client.

8. The HLR returns an acknowledgement to the new VLR. The MCL table of the new VLR is inserted a record. If the mobile client has never visited this VLR, whose three attribute values are respectively $\mathrm{MC}_{i} d$, "Latest LA", and NULL. Otherwise. Only updates the Type and the Pointer values of its record.

9. The HLR updates its data about the mobile client's new location and informs the old LA about the movement of the mobile client.

10. The mobile client's record in the MCL table of the old LA is updated. Its second and third attribute values are modified to the "Visited LA" and the new LA's location. Respectively. The old LA also sends an acknowledgement message to the HLR.

11. The old LA informs the old VLR of location change of the mobile client.

12. The mobile client's record in the old VLR's MCL table is updated. Its second and third attribute values changed to "Visited VLR" and the location of the old LA, respectively. The old VLR also sends an acknowledgement to the old LA.

13. End of the Location Registration. 


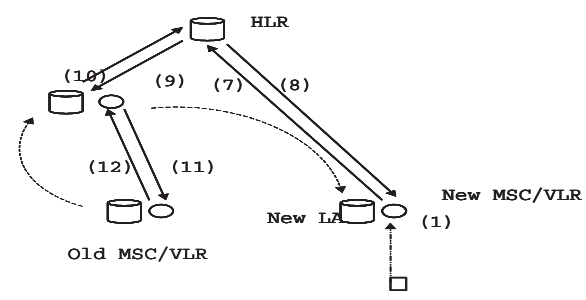

Fig. 1. The case which the new VLR informs the HLR

\subsection{Call Tracking}

Figure 2 are a example that could occur while locating the callee. The details of the operation is as follows.

1. When a VLR receives a request of locating a callee, it first checks whether its MCL table has the callee's record. If yes, then sends the locating requite to the LA stated in this record. Otherwise, jump to Step 7.

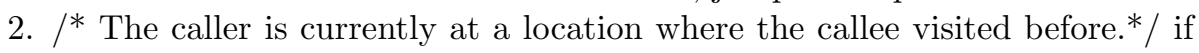
the record of the MCL table of the LA stated in Step 1 says that this LA is a "Visited LA", then goto Step 3. If it says "Latest LA", goto Step 4.

3. The Locating request is forwarded to this visited LA (following the pointer given in the record of Step 2). While the request is forwarded to the next LA, the callee's record is again searched from this LA's MCL table. Goto Step 2.

4. The latest LA (who is overseeing the callee) finds the callee's record from the MCL table. If the value of the Pointer field is NULL, then the callee is right in one of this LA's governing Ras. Hence, a message is forwarded to the caller's VLR to make the connection. Goto step 13. If the value of the Pointer field is not NULL, then it must be a VLR who is currently overseeing the callee. Hence, the locating request is sent to the latest VLR to which the Pointer field refers.

5. The latest VLR sends a message to the caller's VLR to make a connection.

6. Goto Step 13

7. $/ *$ The caller is at a location where the callee has not visited before. Updates of the callee's new location in the LA, VLR, and HLR are associated with this locating operation */

8. The HLR forwards the request to the callee's LA.

9. The callee's latest LA forwards the request to the latest VLR. Also, the callee's record in this VLR's MCL table is updated by replacing its type with "Latest LA" and Pointer with NULL.

10. The callee's VLR acknowledges the receipt of the message to the LA and the LA will then update the callee's record in its MCL table by replacing type with "Visited LA" and Pointer with a pointer to the callee's current residing VLR. 
11. the callee's VLR sends a message to the GLR. The HLR updates the callee's new location to the new latest LA (i.e., the callee's current VLR).

12. The HLR forwards the message about the current location (VLR) of the callee to the caller's VLR and the connection between the caller's VLR and the callee's VLR is built.

13. End of the Call Tracking Operation.

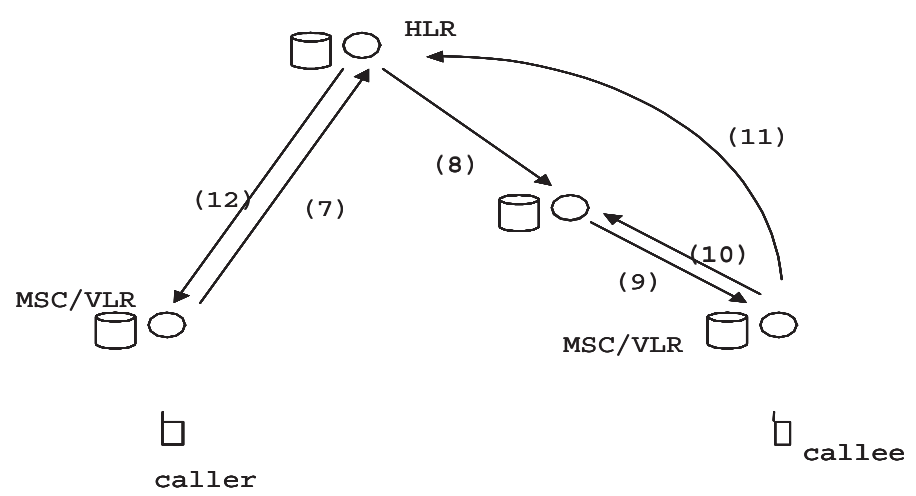

Fig. 2. The case of locating through the HLR

\section{Traffic Cost Model}

We present the traffic cost models that used to evaluate the performance of the proposed strategy.

The parameters used in our cost models are listed in Table 1 . The costs of the IS-41 strategy, was discussed in the literature [4] [8]. In order to make a fair and reasonable comparison, we make some general assumptions and based on which we derive their cost functions in a uniform way. As the local database processing cost is insignificant comparing to the long communication time, we only consider communication cost in this derivation. Communication cost is dependent on the "distance" between two parties, and is classified into three levels: two parties are under different RSTPs, two parties are under the same RSTP but different LSTPs, and two parties under the same LSTP. Their costs are respectively $\mathrm{h} 1, \mathrm{~h} 2$ and $\mathrm{h} 3$, we also need to use probability to model the location distribution of two communicating parties.

\subsection{Cost Functions}

The tasks of location management include managing registration operations and call tracking operations. Hence, the location management cost is computed 
Table 1. The symbols of the parameters and their meanings

\begin{tabular}{|c|l|}
\hline \multicolumn{2}{|c|}{ System Parameters } \\
\hline Symbol & Meaning \\
\hline$h_{1}$ & $\begin{array}{l}\text { The cost of sending a message from VLR to another VLR under } \\
\text { a different RSTP; }\end{array}$ \\
\hline$h_{2}$ & $\begin{array}{l}\text { The cost of sending a message from VLR to another VLR under } \\
\text { a different LSTP but the same RSTP; }\end{array}$ \\
\hline$h_{3}$ & $\begin{array}{l}\text { The cost of sending a message from VLR to another VLR under } \\
\text { the same LSTP; }\end{array}$ \\
\hline$P_{L}$ & $\begin{array}{l}\text { The probability of a mobile client's moving into a new RA which is under } \\
\text { the same LSTP as the last RA that the client just left; }\end{array}$ \\
\hline$P_{R}$ & $\begin{array}{l}\text { The probability of a mobile client's moving into a new RA which is under } \\
\text { the same RSTP as the last RA that the client just left; }\end{array}$ \\
\hline$C M R$ & The call-to-mobility ratio; \\
\hline
\end{tabular}

according to these two operations. As the ratio of the number of calls (locating a callee) to mobility and defined as

$$
\text { total cost }=\frac{1}{C M R} \cdot \text { Registration cost }+ \text { Calltracking cost }
$$

where CMR is the Call-to-Mobility Ratio.

We will use this definition in calculating the total cost of each strategy. For each operation, the time (cost) is counted from the instant that the VLR of the mobile client submits a request is finished. The cost of an operation that is not directly related to the locating operation is not counted in our cost functions. For instance, in the IS-41 scheme the location operation is counted from the time the client's new arriving VLR sending a request to the HLR till the time this VLE receives a message from the HLR to indicate that the location of the client has been update in the HLR. This is the cost of a location operation in the IS-41 scheme. Other operations such as sending a message from the HLR to the former VLR to remove the obsolete record from its table are not counted in our cost models. This is mainly because these operations are not as urgent and can be executed at a later time. Note that ignoring such a cost in our model is for reason of understanding the effect of other key steps in an operation. All such operations in different strategies are treated in the same way.

\subsection{Cost Functions of the IS-41 Strategy}

The IS-41 strategy is a standard scheme and is often used to be compared with other strategy. Hence, we first present the cost functions of the IS-41 strategy. Based on the definition of total cost, the total cost $C_{\text {Basic }}^{\text {total }}$ of the IS-41 strategy can be represented as follows.

$$
C_{I S-41}^{\text {total }}=\left(\frac{1}{C M R}\right) \cdot C_{I S-41}^{R}+C_{I S-41}^{T}
$$


For clearness reason, we list the steps of the IS-41 strategy in the following for a reference. Its idea has been discussed in Section 2.The registration cost of the IS-41 strategy is therefore.

$C_{I S-41}^{R}=2 \cdot h_{1}$

In this algorithm, the call tracking cost of the strategy becomes $C_{I S-41}^{T}=4 \cdot h_{1}$

\subsection{Cost Functions of the DCC Strategy}

We derive the total cost of the DCC strategy. Again, total cost can be stated as follows:

$C_{D C C}^{\text {total }}=\left(\frac{1}{C M R} \cdot C_{D C C}^{M}+C_{D C C}^{L}\right.$

The algorithm of the DCC strategy is the same as that of the Dynamic LA strategy. That is,

$$
C_{D C C}^{M}=\left\{\begin{array}{lr}
\left(P_{R} \cdot h_{2}+P_{L} \cdot h_{3}\right)+\frac{1}{2} \cdot\left(h_{2}+h_{3}\right) & \text { if } \frac{1-P_{L}-P_{R}}{C M R}<1 \\
2 \cdot h_{1} & \text { if } \frac{1-P_{L}-P_{R}}{C M R} \geq 1
\end{array}\right.
$$

As for the location cost, there is a subtle the DCC strategies in computing the cost. Notice that in the DCC strategy wherever a client moves out of the range of a RSTP, the new VLR (that the client just entered) becomes the new LA (an update). Updating a new latest LA makes the distance between two linked LAs much shorter than that in the previous strategy, and so does the communication cost, This factor is considered in the cost model.Two cases are considered.

1. Locate the callee through HLR.

2. Locate the callee througha VLR where the callee visited before.

As for case 2, its cost also involves four tasks (a), (b), (c), and (d). The costs of (a) and (c) of this strategy, however, are both $\frac{1}{2} \cdot\left(h_{2}+h_{3}\right)$ The factor of $\mathrm{h}_{1}$ is not in this cost, because the LA and its linked VLR will always be in the same RSTP. The cost of $(\mathrm{d})$ is still $\frac{1}{3}\left(h_{1}+h_{2}+h_{3}\right)$. Computing the cost of (b) involves two cases:

- Two linked LAs belong to different RSTPs: Between two consecutive calls, a mobile client makes on average $\frac{1}{C M R}$ moves. As the region of a RSTP is rather large, we assume that the callee will at most move out of the region of a RSTP once between two consecutive calls/ The other $\left(\frac{1}{C M R}-1\right)$ moves are all within the same RSTP. That is, the cost of this case is

$$
\left(1-\left(\frac{1}{C M R} \cdot\left(P_{L}+P_{R}\right)^{\frac{1}{C M R}-1} \cdot\left(1-P_{L}-P_{R}\right)\right) \cdot h_{1}\right.
$$

- Two linked LAs belong to the same RSTP: The probability of this case is 1-(the above case) which is $\left(1-\left(\frac{1}{C M R} \cdot\left(P_{L}+P_{R}\right)^{\frac{1}{C M R}-1} \cdot\left(1-P_{L}-P_{R}\right)\right)\right.$ For two LAs within the same RSTP, as they may be either within the same LSTP or different LSTPs, we simply take the average in computing their communication cost, which is $\frac{1}{2} \cdot\left(h_{2}+h_{3}\right)$ Finally, we have the total cost 


$$
\begin{aligned}
& C_{D C C}^{L}=P+Q, \text { where } \\
& P=\left(1-\sum_{i=0}^{K_{C \& C}} P_{i}\right) \cdot C_{D L A}^{L} \\
& Q=\left(\sum_{i=0}^{K_{C \& C}} P_{i}\right) \cdot\left\{\frac { 1 } { K _ { C \& C } } \cdot \sum _ { i = 0 } ^ { K _ { C \& C } } \left[\frac{1}{2} \cdot\left(h_{2}+h_{3}\right)\right.\right. \\
& +i \cdot\left[\left(1-\frac{1}{C M R} \cdot\left(P_{L}+P_{R}\right)^{\frac{1}{C M R}}-1 \cdot\left(1-P_{L}-P_{R}\right)\right) \cdot\left(\frac{1}{2} \cdot\left(h_{2}+h_{3}\right)\right)\right. \\
& \left.+\left(\frac{1}{C M R} \cdot\left(P_{L}+P_{R}\right)^{\frac{1}{C M R}}-1 \cdot\left(1-P_{L}-P_{R}\right)\right) \cdot h_{1}\right]+\frac{1}{2} \cdot\left(h_{2}+h_{3}\right) \\
& \left.+\frac{1}{3} \cdot\left(h_{1}+h_{2}+h_{3}\right)\right\}
\end{aligned}
$$

\section{Performance Analysis}

We see two important factors that affect the performance of the DCC strategy: $K_{C \& C}$ and $P_{i}$ where $K_{C \& C}$ is the length of a forwarding and $P_{i}$ is the probability that a caller's request is issued from $L A_{i}$ and its overseeing VLRs. Hence, we first study the effect of these factor to the DCC strategies. Also, we vary the ratio h1/ h3, which represents varying region size of a RSTP versus a LSTP. This is a general factor which affects all strategies. The default values of the parameters used in our evaluation are given Table 2.

Table 2. Default values of parameters

\begin{tabular}{|c|c|c|c|c|c|c|c|c|}
\hline Parameter & $\mathrm{h}_{1}$ & $\mathrm{~h}_{2}$ & $\mathrm{~h}_{3}$ & $\mathrm{P}_{L}$ & $\mathrm{P}_{R}$ & $\mathrm{CMR}$ & $\mathrm{k}_{\text {dc\&c }}$ & $\mathrm{P}_{i}$ \\
\hline Default Value & 8 & 4 & 1 & 0.7 & 0.2 & 0.5 & 6 & 0.05 \\
\hline
\end{tabular}

\subsection{The Cost Result of $P_{i}$}

$P_{i}$ is the probability that a caller places a call from a VLR that happens to be under one of the linked LAs of the callee. When this occurs, the locating cost is cheap. We vary $P_{i}$ from 0.01 to 0.16 . As the default $K_{C \& C}$ is 6 , the total probability of a call from the VLR under a linked LAs (i.e., $\left.\sum_{i=1}^{K_{C \& C}} P_{i}\right)$ is actually $0.06 \sim 0.96$ The result given in Figure 3 shows. However, for the $C \& C$ strategies a dramatic decrease of the cost when $P_{i}$ increases. Although in general $P_{i}$ may not be large for every kind of mobile clients, it could definitely be so for a certain type of users. For instance, most of the commuters always drive along the same route between their offices and houses. Many office workers and students, etc., also appear at several fixed location regularly. Our performance result shows that the proposed strategy is especially good for managing mobile clients of this kind. The curves of the other strategies are flat because they are independent of the varying parameter $P_{i}$. 


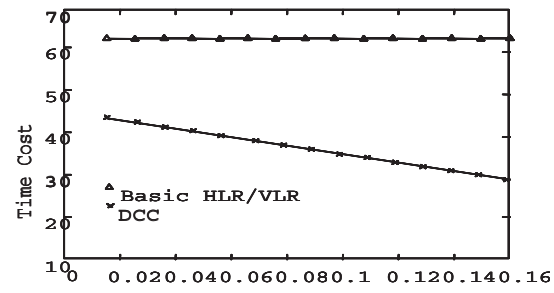

Fig. 3. $\mathrm{P}_{i}$ to the IS-41 strategies

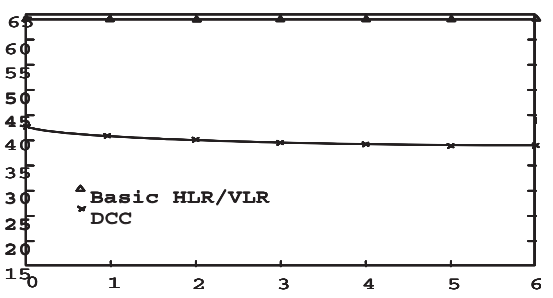

Fig. 4. $4 \mathrm{~K}_{D C C}$ to the DCC strategy

\subsection{The Cost Result of $K_{D C C}$}

In our proposed strategy, multiple LAs may be liked together due to the movement of a mobile client. The length of this link is represented by $K_{D C C}$. Figure 8 shows the result by varying $K_{D C C}$. A large $K_{D C C}$ means that many VLRs that are under the linked LAs can locate a callee through the providing links of LAs, which helps to reduce the locating cost. However, the curves of the proposed strategy will become high after a certain $K_{D C C}$ point. This is because the locating cost will increase with the lengthy linked LAs. The longer the linked LAs, the longer it takes to locate a callee. According to our derivation earlier, the length of the link should be less than $\left[4{ }^{*} h_{1} / c_{1}-3\right]$ in order to have benefit. The improvement of the proposed strategy over the IS-41 strategy is very significant.

The reason is the links in the proposed strategy are built on LAs. While a mobile client moves, he/she may move out of the boundary of a VLR easily and get into another VLR. But in the proposed strategy, the client doe not move out of the boundary of a LA so easily because the region of a LA could be much larger than that of a VLR.

\section{Conclusions}

In this paper we proposed a new mobility strategy for reducing location traffic cost in mobile networks. Different from the previous strategies, the proposed strategy can reduce the locating cost at the same time. The proposed strategy avoids updating the client's location to the HLR when the client moves to a new VLR. The client's new VLR always updates the client's location to the LA. We also derived the cost models of the proposed strategies and the related work. Our analysis results reveal that in most case the proposed strategy performs better than the IS-41 strategies. This is mainly because it can reduce the locating cost simultaneously, while the other strategies only reduces one of them. Our strategies are also easy to implement based on mobile computing environment. 


\section{References}

1. Amotz Bar-Noy and Ilan Kessler, "Tracking Mobile Users in Wireless Communication Networks", IEEE Transactions on Information Theory, Vol. 39, No. 6, 1993, pp. $1877-1886$.

2. Ing-Ray Chen, Tsong-Min Chen, and Chiang Lee, "Modeling and Analysis of Forwarding and Resetting Strategies for Location Management in Mobile Environments", in Proceedings of the 1996 International Computer Symposium (ICS‘96), Kaohsiung, Taiwan, R.O.C., December 1996, pp. 121-128.

3. Ing-Ray Chen, Tsong-Min Chen, and Chiang Lee, "Performance Characterization of Forwarding Strategies in Personal Communication Networks", in 21th IEEE International conferences of Computer Software and Application (COMPSAC'97), Washington, D.C., August 1997, pp. 137-142.

4. Ing-Ray Chen, Tsong-Min Chen, and chiang Lee, "Performance Evaluation of Forwarding Strategies for Location Management in Mobile Networks", The Computer Journal, Vol. 41, No. 4, August 1998, pp. 243-253.

5. Ing-Ray Chen, Tsong-Min Chen, and Chiang Lee, "Analysis and Comparison of Location Strategies for Reducing Registration Cost in CS Networks", Wireless Personal Communications journal, Vol. 12, No. 2, 2000, pp. 117-136.

6. EIA/TIA IS-41.3, "Cellular Radio Telecommunications Intersystem Operations", Technical Report, Technical Report (RevisionB), July 1997.

7. Joseph S. M. Ho and Ian F. Akyildiz, "A Dynamic Mobility Tracking Policy for Wireless Personal Communications Networks", In IEEE Global Telecommunications Conference (GLOBECOM‘95), Singapore, November 1995, pp. 1-5.

8. Joseph S. M. Ho and Ian F. Akyildiz, "Local Anchor Scheme for Reducing Signaling Costs in Personal Communications Networks", IEEE/ACM Transactions on Networking, Vol. 4, No. 5, October 1996, pp. 709-725.

9. T. Imielinski and B. R. Badrinath, "Querying in Highly Mobile Distributed Environments", in Proceedings of the 18th VLDB Conference, Vancouver. British Columbia. Canada, 1992, pp. 41-52.

10. T. Imielinski and B. R. Badrinath, "Mobile Wireless Computing: Challenges in Data Management", Communications of the ACM, Vol. 37, No. 10, 1994, pp. 1828.

11. Ravi Jain, Yi-Bing Lin, Charles Lo, and Seshadri Mohan, "A Caching Strategy to Reduce Network Impacts of PCS", EEEE Journal on Selected Ares in Communications. Vol. 12, No. 8, October 1994, pp. 1434-1444. 